\title{
The downregulated long noncoding RNA DHRS4-ASI is protumoral and associated with the prognosis of clear cell renal cell carcinoma
}

This article was published in the following Dove Press journal:

OncoTargets and Therapy

\section{Changlin Wang, I,* Gang Wang, ${ }^{1, *}$ Zijian Zhang, ${ }^{1, *}$ Zichun Wang,' Minghua Ren,' Xiaoxiong Wang, ${ }^{2}$ Haoming Li,' Yipeng Yu,' Jing Liu,' Licheng Cai,' Yong $\mathrm{Li}^{3}{ }^{3}$ Daming Zhang, ${ }^{2}$ Cheng Zhang'}

'Department of Urology, The First Affiliated Hospital of Harbin Medical University, Harbin, Heilongjiang, China; ${ }^{2}$ Department of Neurosurgery, The First Affiliated Hospital of Harbin Medical University, Harbin, Heilongjiang, China; ${ }^{3}$ Department of PET/CT, The First Affiliated Hospital of Harbin Medical University, Harbin, Heilongjiang, China

*These authors contributed equally to this work
Correspondence: Cheng Zhang Department of Urology, The First Affiliated Hospital of Harbin Medical University, 23 Youzheng Street, Nangang District, Harbin I5000I, Heilongjiang, China

Tel +86 I38 36I8 2568

Email doctorcheng77@।63.com

Daming Zhang

Department of Neurosurgery, The First Affiliated Hospital of Harbin Medical University, 23 Youzheng Street, Nangang District, Harbin I5000I, Heilongjiang, China

Tel +86 I364460604 I

Email zhangdaming@ymail.com
Background: Long noncoding RNAs (lncRNAs) have been identified as important factors in cancer biology and are deregulated in many cancers. The present study aimed to determine the expression and roles of IncRNA DHRS4-AS1 in the progression of clear cell renal cell carcinoma (ccRCC).

Methods and results: Using high-throughput RNA-sequencing data of ccRCC tumors from the Cancer Genome Atlas project, we identified lncRNA DHRS4-AS1 as significantly associated with ccRCC patients' overall survival. We confirmed the downregulation of DHRS4-AS1 in ccRCC by assessing its expression levels in a cohort of 52 tumor and paired non-tumor samples. In addition, we found that low DHRS4-AS1 expression was significantly associated with a high tumor node metastasis stage, lymph node metastasis, advanced pathological grade and poor prognosis. Furthermore, DHRS4-AS1 overexpression inhibited the progression of cell cycles of ccRCC in vitro. These data indicate that DHRS4-AS1 functions by preventing the proliferation and invasion, inhibiting the cell cycle progression and promoting the apoptosis of ccRCC cells.

Conclusion: Taken together, our findings identify the role of DHRS4-AS1 as a tumor inhibitor in ccRCC for the first time, demonstrating that DHRS4-AS1 is a potential prognostic biomarker that could potentially be applied in ccRCC therapy.

Keywords: long noncoding RNAs, DHRS4-AS1, clear cell renal cell carcinoma, prognosis

\section{Introduction}

Renal cell carcinoma (RCC) is one of the most common kidney carcinomas, which is the sixth leading cause of death in the USA. The male to female patient ratio is 1.6:1.0, with patient ages ranging from 40 to 70 years at diagnosis. ${ }^{1}$ Clear cell renal cell carcinoma (ccRCC) is a major type of renal epithelial malignancies and is responsible for approximately $70 \%-80 \%$ of all RCC diagnoses. ${ }^{2}$ It originates from the renal proximal tubule with a histological characteristic of clear cytoplasm with incidental eosinophilia areas. Surgical excision is considered the primary treatment method for ccRCC, by which most ccRCC patients will be cured. However, approximately $30 \%$ of new ccRCC cases show evidence of local or distant metastasis associated with poor prognosis, with a median survival time of only 13 months. ${ }^{3-5}$ Therefore, it is necessary to identify sensitive and specific ccRCC targets and to develop novel therapeutic strategies for ccRCC.

Long noncoding RNAs (lncRNAs) are a newly discovered kind of noncoding RNAs (ncRNAs) that are defined as RNA transcripts longer than 200 nucleotides and are not or only partially translated into proteins. ${ }^{6}$ Recent studies have suggested that 
lncRNAs play crucial roles in a range of biological processes, including cell proliferation, apoptosis and differentiation, and in cancer migration and metastasis. ${ }^{7-12}$ Numerous dysregulated lncRNAs have been identified in various tumors. HOXA-AS2 promotes breast cancer proliferation and invasion by manipulating miR-520c-3p expression. ${ }^{13}$ XIST promotes pancreatic cancer proliferation by modulating the miR-133a/EGFR axis. ${ }^{14}$ Moreover, several kinds of $\operatorname{lncRNAs}$ have been reported as new potential survival predictors for cancer patients, such as HOTAIR, HOTTIP, MALAT1 and CCAT1. ${ }^{15-18}$ Recently, some studies have reported certain lncRNAs as prognostic biomarkers for ccRCC. For example, decreased expression levels of lnc-ZNF180-2, CADM1-AS1 and TCL6 are correlated with a worse prognosis in ccRCC patients in terms of shorter progression-free survival and cancer-specific survival. ${ }^{19-21}$ However, more valuable, potential lncRNA biomarkers and their molecular mechanisms need to be identified to improve the clinical prognosis of ccRCC patients.

DHRS4 antisense RNA 1 (DHRS4-AS1) is a 3,052-bp spliced and polyadenylated noncoding RNA transcribed from intron 1 of the DHRS4 gene in the antisense direction that controls the local epigenetic status of each gene promoter within the DHRS4 gene cluster in a discriminatory manner. ${ }^{22,23}$ In our study, we found reduced expression levels of DHRS4-AS1 in ccRCC tissues compared with adjacent non-tumor tissues and that the expression levels of DHRS4$A S 1$ were inversely related to clinicopathological features and ccRCC patients' prognosis. Moreover, consistent with in vitro results, we demonstrated that $D H R S 4-A S 1$ played a critical role in diminishing ccRCC cell proliferation, migration and invasion during ccRCC progression by a series of in vitro assays. Our results suggest that lncRNA DHRS4-AS1 could represent a new indicator of poor prognosis and may be a potential novel therapeutic target for ccRCC patients.

\section{Materials and methods Clinical samples and cell culture}

In this study, fresh tumor and matched adjacent normal tissue samples were collected from patients who underwent radical nephrectomy or nephron-sparing surgery between 2012 and 2017 in the First Affiliated Hospital of Harbin Medical University. None of the patients received chemotherapy or radiotherapy prior to surgery. The clinicopathological information was obtained from patients' history record including patient age, overall survival duration, tumor cell differentiation, T category, clinical disease stage and lymph node status. All ccRCC cases were confirmed by a senior pathologist, samples were staged according to the tumor node metastasis (TNM) classification and criteria of the World Health Organization (WHO), and tumor grade was assessed in accordance with the WHO criteria. All tissue samples were immediately stored in liquid nitrogen until use. This study protocol conformed to clinical research guidelines and was approved by the research ethics committee of the First Affiliated Hospital of Harbin Medical University. Written informed consent was obtained from all patients who participated in this study. Cell lines 786-O, 769-P, HK-2 and 293T were purchased in 2016-2017 from the Chinese Academy of Science Committee Type Culture Collection Cell Bank (Shanghai, People's Republic of China). Two ccRCC cell lines, 786-O and 769-P, were cultured in RPMI-1640 (Thermo Fisher Scientific, Waltham, MA, USA) medium supplemented with $10 \%$ fetal bovine serum (FBS). Normal renal cell line HK-2 and 293T cells were cultured in DMEM (Thermo Fisher Scientific) supplemented with $10 \%$ FBS.

\section{Bioinformatics analysis databases}

The ccRCC patients' clinical and RNA-Seq data were obtained from The Cancer Genome Atlas project (TCGA) (https://tcga-data.nci.nih.gov/). ${ }^{24}$ The expression of lncRNAs was quantified by a customized data analysis pipeline that included the steps of quality control, alignment and expression quantification. The DHRS4-AS1 methylation data were obtained from University of California Santa Cruz (UCSC) (http://genome.ucsc.edu/). A gene sets enrichment analysis was performed using Gene Set Enrichment Analysis (GSEA) software (http://software.broadinstitute.org/gsea/index.jsp) with the MSigDB C2 CP: KEGG gene sets collection (186 gene sets available). Gene sets with a false discovery rate (FDR) value $<0.01$ after performing 1,000 permutations were considered significantly enriched. ${ }^{25}$

\section{RNA extraction and quantitative real-time polymerase chain reaction ( $q R T-P C R$ )}

Total RNA was isolated by TRIzol reagent (Thermo Fisher Scientific, Carlsbad, CA, USA) according to the manufacturer's instructions. One $\mu \mathrm{g}$ of total RNA was reversetranscribed into cDNA using High-Capacity cDNA Reverse Transcriptase Kits (Toyobo, Osaka, Japan). The level of DHRS4-AS1 relative to the control gene, GAPDH, was determined by qRT-PCR using a Lightcycler-480II (Hoffman-La Roche Ltd., Basel, Switzerland). The PCR conditions were as follows: $95^{\circ} \mathrm{C}$ for $10 \mathrm{~min}, 40$ cycles of $95^{\circ} \mathrm{C}$ for $15 \mathrm{~s}$, and $60^{\circ} \mathrm{C}$ for $60 \mathrm{~s}$. PCR amplification was performed in triplicate. Changes in threshold cycle (CT) values were calculated by the CT $\left(2^{-\Delta \Delta \mathrm{CT}}\right)$ method. 


\section{Lentiviral construction and production}

Synthesized full-length DHRS4-ASI (NR_023921.2) was directly cloned into a pLVX vector through EcoRI and BamHI using an In-Fusion Cloning kit (Clontech, Beijing, China). Lentiviral packaging was performed in 293T cells. Briefly, 293T cells were transiently transfected with pLVX plasmid and the packaging plasmids pLP1, pLP2 and pLP/ VSVG using a calcium phosphate-based transfection method. Supernatant containing lentiviral particles was harvested 3 days post-transfection and was centrifuged at 2,000 rpm for $10 \mathrm{~min}$ and filtered through a $0.45-\mu \mathrm{m}$ filter to remove cellular debris. Then, the crude lentivirus was concentrated by a Lenti-X concentration kit (Clontech). The purified lentivirus was stored at $-80^{\circ} \mathrm{C}$.

\section{Demethylation treatment using 5-Aza-2'-deoxycytidine (Aza)}

Treatment of RCC cell line using Aza was added to the medium. ${ }^{26}$ Briefly, cells were treated with 4,8 and $16 \mu \mathrm{M}$ Aza (MedChemExpress, Monmouth, NJ, USA) for $72 \mathrm{~h}$ and harvested for RNA extraction, DMSO was added to cells as the control experiment.

\section{Cell proliferation assay}

A cell proliferation assay was carried out using the Cell Counting Kit-8 (CCK-8) according to the manufacturer's instructions (CCK-8; Dojindo Molecular Technologies, Inc., Kyushu, Japan). Briefly, cells were seeded in a 96-well plate at $3 \times 10^{3}$ cells per well. Cell proliferation was assessed after culturing for 24,48 and $72 \mathrm{~h}$. After adding $10 \mu \mathrm{L}$ of CCK-8 reagents per well and incubating at $37^{\circ} \mathrm{C}$ for $2 \mathrm{~h}$, the absorbance was measured at $450 \mathrm{~nm}$ using a microplate reader (BioTek, Winooski, VT, USA).

\section{Colony formation assay}

For the colony formation assay, cells were seeded in six-well plates at 500 cells per well and cultured in RPMI-1640 with $10 \%$ FBS for 10 days. Colonies were fixed with $4 \%$ formaldehyde and stained with $0.1 \%$ crystal violet. Colonies with a diameter greater than $1 \mathrm{~mm}$ were counted.

\section{Determination of cell apoptosis and cell cycle by flow cytometry}

Apoptosis was analyzed with the Annexin V-PE Apoptosis Detection Kit (BD Biosciences, San Jose, CA, USA) according to the manufacturer's instructions. Briefly, cells were washed and resuspended in $1 \times$ binding buffer. Then, $100 \mu \mathrm{L}$ of the cell solution $\left(1 \times 10^{5}\right.$ cells $)$ was mixed with $5 \mu \mathrm{L}$ of Annexin V-PE and $5 \mu \mathrm{L}$ of 7-AAD and the cells were kept on ice for $15 \mathrm{~min}$ in the dark. Next, $400 \mu \mathrm{L}$ of $1 \times$ binding buffer was added to each tube for immediate analysis by flow cytometry (Becton Dickinson, Mountain View, CA, USA). The cell cycle was evaluated by flow cytometry using the Cycle TEST ${ }^{\mathrm{TM}}$ PLUS DNA Reagent Kit (BD Biosciences). Briefly, indicated numbers of cells were plated in 6-well plates. The cells were collected and centrifuged at $400 \times g$ for $5 \mathrm{~min}$. Then, $250 \mu \mathrm{L}$ of Solution A (trypsin buffer) was added to each tube and gently mixed by tapping the tube by hand, allowing Solution A to react for $10 \mathrm{~min}$ at room temperature. Next, $200 \mu \mathrm{L}$ of Solution B (trypsin inhibitor and RNase buffer) was added to each tube, followed by incubation for $10 \mathrm{~min}$. Then, $200 \mu \mathrm{L}$ of cold $\left(2^{\circ} \mathrm{C}-8^{\circ} \mathrm{C}\right)$ Solution $\mathrm{C}$ (propidium iodide stain solution) was added to each tube, and then the tubes were incubated for $10 \mathrm{~min}$ in the dark on ice. The cells were analyzed by flow cytometry (Becton Dickinson). Each assay was carried out triplicate.

\section{Wound-healing assay}

Cells were seeded in 24 -well plates at $1 \times 10^{5}$ cells per well and incubated at $37^{\circ} \mathrm{C}$ in $5 \% \mathrm{CO}_{2}$ for $24 \mathrm{~h}$. To reduce cell proliferation, the cells were maintained in serum-free medium throughout the experiment. An approximate $0.4-0.5-\mathrm{mm}$ line was scraped using the fine end of sterile pipette tips. Then, the plate was washed with $1 \times$ PBS three times. Images were captured using an inverted microscope and analyzed using ImageJ software (Rawak Software Inc., Stuttgart, Germany).

\section{Transwell assay}

Briefly, approximately $1 \times 10^{5}$ cancer cells were suspended in $150 \mu \mathrm{L}$ serum-free medium added to the upper chamber and a total of $600 \mu \mathrm{L}$ medium with $10 \%$ serum was added to the bottom chamber as a chemoattractant. Cells were seeded in the 24-well Boyden chamber $(8 \mu \mathrm{m}$; Corning Incorporated, Corning, NY, USA) and incubated for $24 \mathrm{~h}$. After wiping off the non-migrating cells in the upper chamber attentively with cotton swabs, the cells on the polycarbonate membranes were fixed with $4 \%$ paraformaldehyde and stained with crystal violet. The cells were counted in five random fields on each membrane.

\section{Western blot}

Cells were lysed to obtain proteins using radio immunoprecipitation assay. Proteins were separated by $12.5 \%$ or $10 \%$ sodium dodecyl sulfate polyacrylamide gel electrophoresis and then transferred to nitrocellulose filter membrane (GE Amersham, Germany). After blocking in 5\% non-fat milk, the membrane was immunoblotted overnight at $4^{\circ} \mathrm{C}$ with primary antibodies: rabbit monoclonal antibody against human (1:1,000 dilution; Wanleibio, Beijing, China) and $\beta$-actin 
(1:5,000 dilution; Sigma-Aldrich Co., St Louis, MO, USA). A secondary antibody, horseradish peroxidase-conjugated anti-rabbit IgG antibody (1:2,000 dilution; ZSGB-BIO, Beijing, China), was incubated with the membrane for $1 \mathrm{~h}$ after three washes with TBST. Signals were detected with electricity chemistry lumina detection reagent (Beyotime, Shanghai, China). The images were obtained by Quantity One (Bio-Rad Laboratories Inc., Hercules, CA, USA).

\section{Statistical analysis}

The statistical analysis was performed with GraphPad Prism 7.0 (GraphPad Software Inc., San Diego, CA, USA) and the results are presented as the mean $\pm \mathrm{SD}$ (SD). Chi-square tests were performed to explore the associations between DHRS4-AS1 levels and clinical and pathologic factors. Overall survival was calculated as the time from surgery until the occurrence of death. The Kaplan-Meier method and log-rank test were used to compare the differences between patient groups. Survival data were evaluated using univariate and multivariate Cox proportional hazards models. $P$-values $<0.05$ were considered statistically significant.

\section{Results}

\section{The expression of DHRS4-ASI is} diminished in $\mathrm{CCRCC}$ cell lines and tissues Considering that survival prognosis in patients with ccRCC is extremely variable, we analyzed the high-throughput RNA-sequencing data of the ccRCC cohort from the TCGA database relative to survival prognosis. Seven lncRNAs were analyzed using a univariable Cox regression model and were significantly correlated with the patients' overall survival $(P<0.001$, Table 1$)$. Higher expression levels of three lncRNAs corresponded to shorter survival (LINC00338, $P D X D C 2 P$ and $S N H G 3)$. In contrast, higher expression levels of four lncRNAs (DHRS4-AS1, RP11-815I9.4, RP11$732 M 18.3$ and OIP5-AS1) were associated with longer survival. Because the FDR value is the most significant variable in the DHRS4-AS1 COX analysis, we chose to focus on DHRS4-AS1 in this study. To verify that DHRS4-AS1 is downregulated in ccRCC tumor tissues, we analyzed the expression levels of DHRS4-AS1 in 52 sets of ccRCC tumor tissues and their corresponding adjacent non-tumor tissues in patents from the First Affiliated Hospital of Harbin Medical University (Figure 1A) and the TCGA database (Figure 1B). The relative expression level of DHRS4-AS1 in ccRCC tissues was significantly lower than that in the paired adjacent normal tissues, indicating that DHRS4-AS1 may be involved in the pathogenesis of ccRCC. In the clinical classification, DHRS4-AS1 levels in T1+T2 subjects were lower than those of T3+T4 subjects (Figure 1C and D). Consistent with this, the DHRS4-AS1 expression level was lower in patients with a high pathological stage (Figure $1 \mathrm{E}$ and $\mathrm{F}$ ). Reduced expression levels of DHRS4-AS1 were observed in ccRCC cell lines 786-O and 769-P compared with the normal renal cell line HK-2 (Figure 1G). Together, these data show that DHRS4$A S 1$ expression is suppressed in ccRCC tissues and cells and is related to the clinical classification of ccRCC patients.

\section{Downregulation of DHRS4-ASI is} associated with adverse clinicopathological features and poor prognosis

Next, we examined the potential clinical significance of DHRS4-AS1 in ccRCC. Based on the relative expression levels of DHRS4-AS1 (with the median value as the cutoff) in cancer/adjacent tissues, patients were divided into two groups: the DHRS4-AS1 high expression group ( $\mathrm{n}=26)$ and the DHRS4-AS1 low expression group (n=26). The correlation between DHRS4-AS1 expression and clinicopathological characteristics is shown in Table 2. Low DHRS4-AS1 expression was positively associated with present lymph node metastasis $(P=0.048)$, high TNM stage $(P=0.025)$ and advanced pathological grade $(P=0.032)$. The expression level of DHRS4-AS1 was not significantly associated with gender or age at surgery. Consistent with

Table I Seven IncRNAs significantly associated with overall survival

\begin{tabular}{lllll}
\hline Gene ID & Gene symbol & P-value & FDR & Coefficient \\
\hline ENSG0000002I5256.3 & DHRS4-ASI & $0.000336^{*}$ & 0.003595 & -0.39807 \\
ENSG000000264885.I & RPII-8I5I9.4 & $0.003414^{*}$ & 0.018032 & -0.41359 \\
ENSG000000236537.I & RPII-732MII8.3 & $0.003303^{*}$ & 0.018032 & -1.98674 \\
ENSG000000247556.2 & OIP5-ASI & $0.002565^{*}$ & 0.01716 & $-0.2125 I$ \\
ENSG000002349I2.5 & LINC00338 & $0.00553 I^{*}$ & 0.024402 & 0.29512 \\
ENSG00000I96696.8 & PDXDC2P & $0.004089 *$ & 0.019167 & 0.363749 \\
ENSG00000242I25.2 & SNHG3 & $0.000767^{*}$ & $0.007 \mid 93$ & 0.219547 \\
\hline
\end{tabular}

Note: $* P<0.05$.

Abbreviations: IncRNAs, long noncoding RNAs; FDR, false discovery rate. 
A

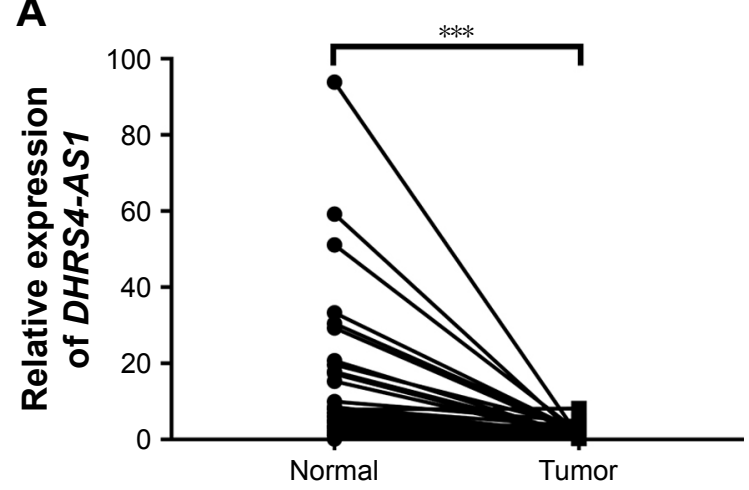

C

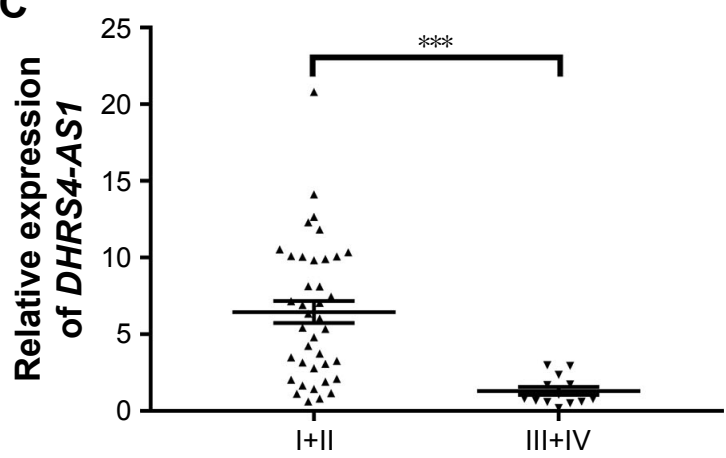

E

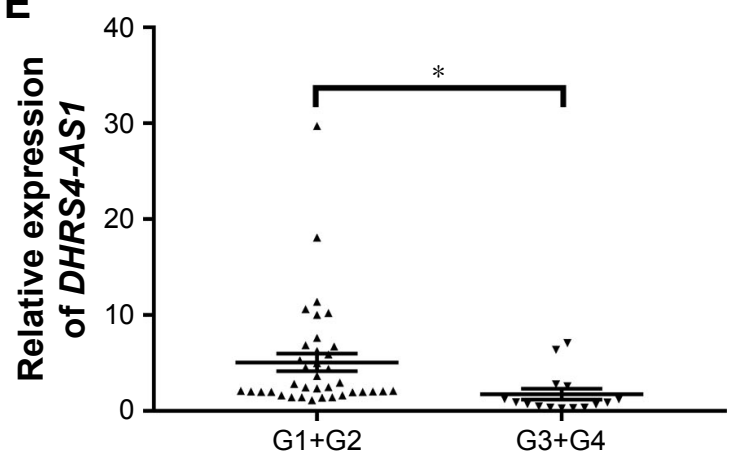

B

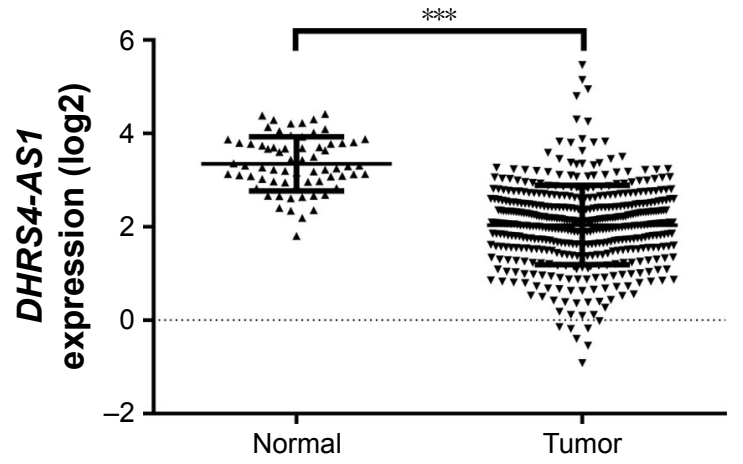

D

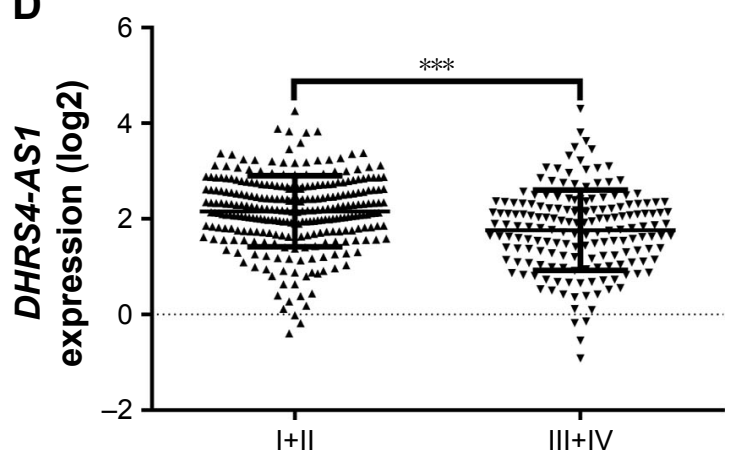

$\mathbf{F}$

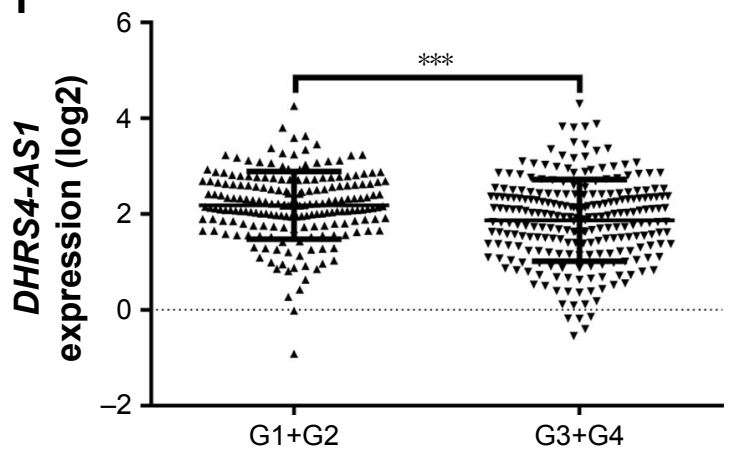

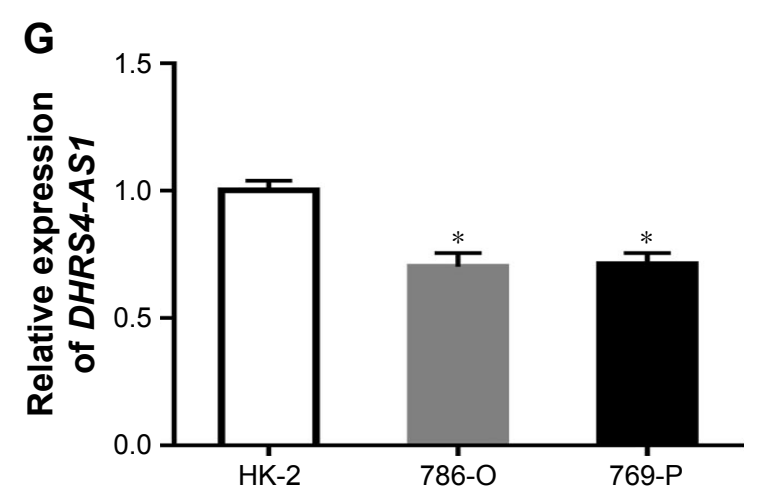

Figure I DHRS4-ASI expression levels in renal cell lines and tissues.

Notes: (A) DHRS4-ASI expression levels were examined by qRT-PCR and normalized to GAPDH expression in 52 paired ccRCC and adjacent normal tissue samples. (B) Relative expression of DHRS4-ASI between ccRCC $(\mathrm{N}=446)$ and normal renal tissues $(\mathrm{N}=67)$ from the TCGA database. (C) Relative expression of DHRS4-ASI between TNM Stages I+II $(\mathrm{N}=39)$ and III+IV (N= I3) tissue samples. (D) Relative expression of DHRS4-ASI between TNM Stages I+II (N=275) and III+IV (N= I7I) tissue samples (TCGA). (E) Relative expression of DHRS4-ASI between Grades I+2 (N=37) and 3+4 $(\mathrm{N}=15)$ tissue samples. (F) Relative expression of DHRS4-ASI between Grades I+2 $(\mathrm{N}=275)$ and $3+4(\mathrm{~N}=17 \mathrm{I})$ tissue samples. $(\mathbf{G})$ Expression levels of DHRS4-ASI in the HK-2 cell line and two ccRCC cell lines were examined by real-time PCR. For all statistical analyses: $* P<0.05$; $* * * P<0.001$. The data are presented as the mean $\pm S D$. Experiments were performed three times.

Abbreviations: DHRS4-ASI, DHRS4 antisense RNA I; qRT-PCR, quantitative real-time PCR; GAPDH, glyceraldehyde 3-phosphate dehydrogenase; ccRCC, clear cell renal cell carcinoma; TCGA, The Cancer Genome Atlas; TNM, tumour node metastasis. 
Table 2 Associations of DHRS4-ASI expression with clinicopathological factors

\begin{tabular}{|c|c|c|c|c|c|}
\hline \multirow[t]{2}{*}{ Characteristics } & \multirow{2}{*}{$\begin{array}{l}\text { Number } \\
\text { of patients }\end{array}$} & \multicolumn{2}{|l|}{ DHRS4-ASI } & \multirow[t]{2}{*}{$\chi^{2}$} & \multirow[t]{2}{*}{$P$-value } \\
\hline & & $\begin{array}{l}\text { High } \\
\text { expression (\%) }\end{array}$ & $\begin{array}{l}\text { Low } \\
\text { expression (\%) }\end{array}$ & & \\
\hline Gender & & & & 0.08 & 0.777 \\
\hline Male & 31 & $15(57.69)$ & $16(61.54)$ & & \\
\hline Female & 21 & II (42.3I) & $10(38.46)$ & & \\
\hline Age (years) & & & & 0.391 & 0.532 \\
\hline$\leq 65$ & 38 & $20(76.92)$ & $18(69.23)$ & & \\
\hline$>65$ & 14 & $6(23.08)$ & $8(30.77)$ & & \\
\hline TNM stage & & & & 5.026 & $0.025^{*}$ \\
\hline $\mathrm{I}+\mathrm{II}$ & 39 & $23(88.46)$ & $16(61.54)$ & & \\
\hline III+IV & 13 & $3(11.54)$ & $10(38.46)$ & & \\
\hline Grade & & & & 4.591 & $0.032 *$ \\
\hline Grade $\mathrm{I}+2$ & 37 & $22(84.62)$ & $15(57.69)$ & & \\
\hline Grade $3+4$ & 15 & $4(15.38)$ & II (42.3I) & & \\
\hline Lymph node metastasis & & & & 3.9 & $0.048 *$ \\
\hline No & 40 & $23(88.46)$ & $17(65.38)$ & & \\
\hline Yes & 12 & $3(11.54)$ & $9(34.62)$ & & \\
\hline Distant metastasis & & & & 0 & $\mathrm{I}$ \\
\hline Mo & 48 & $24(92.31)$ & $24(92.31)$ & & \\
\hline MI & 4 & $2(7.69)$ & $2(7.69)$ & & \\
\hline
\end{tabular}

Note: $* P<0.05$.

Abbreviations: DHRS4-ASI, DHRS4 antisense RNA I; TNM, tumour node metastasis.

this, low DHRS4-AS1 expression was also associated with a high TNM stage $(P<0.001)$ and advanced pathological grade $(P=0.014)$ in a large cohort of 446 ccRCC patients retrieved from the TCGA database (Table S1). Low DHRS4$A S 1$ expression was associated with an overall short survival time $(P=0.0255)$ by Kaplan-Meier analysis (Figure 2A). Higher DHRS4-AS1 expression suggested a good prognosis, which further confirmed the results from the large cohort of 446 ccRCC patients retrieved from the TCGA database $(P=$ 0.0027 ) (Figure 2B). Univariate and multivariate Cox proportional hazards analyses showed that DHRS4-AS1, TNM stage and pathological grade were independent prognostic factors for survival in 52 paired ccRCC patient samples (Table 3 ) and in $446 \mathrm{ccRCC}$ patients retrieved from the TCGA database (Table S2). These results suggest that DHRS4-AS1 may be a good diagnostic biomarker for ccRCC. We then used a receiver operating characteristic (ROC) curve to study the diagnostic value of DHRS4-AS1 in distinguishing between ccRCC tissue and adjacent non-tumor tissue. The area under the curve (AUC) was calculated for the ROC curve and the AUC of the expression level of DHRS4-AS1 was 0.801 (Figure 2C), demonstrating that DHRS4-AS1 has good potential as a biomarker.

\section{KEGG pathway analysis for DHRS4-ASI signature target genes}

We explored the biological functions of DHRS4-AS1 in tumorigenesis and progression in ccRCC. According to the prediction of the GSEA database results, the target gene enrichment results of the KEGG pathways in the present study are listed in Figure 3. Pathways for ribosome, proteasome, oxidative phosphorylation, cell cycle, DNA replication, graft versus host disease, systemic lupus erythematosus, primary immunodeficiency, allograft rejection and natural killer cell-mediated cytotoxicity were enriched with the DHRS4-AS1 target genes (Figure 3A). The expression of DHRS4-AS1 was negatively correlated with the expression of two gene sets previously defined in the TCGA KIRC (kidney renal clear cell carcinoma) data set, including one gene involved in the negative regulation of cell cycle genes (Figure 3B) and one involved with DNA replication genes (Figure 3C).

\section{Exogenetic DHRS4-ASI expression suppressed ccRCC cell proliferation and promoted apoptosis in vitro}

To further examine the role of DHRS4-AS1 in ccRCC cell proliferation, we overexpressed the lncRNA using purified lentivirus for Flag control or DHRS4-AS1. IncRNA DHRS4$A S 1$ expression was comparatively lower in 786-O and 769-P cell lines than in the HK-2 cell line (Figure 1G). Therefore, we selected 786-O and 769-P cells for the DHRS4-AS1 overexpression assay and transfection efficiency was confirmed via qRT-PCR analysis (Figure 4A). To determine the effect of DHRS4-AS1 on the proliferation of ccRCC cells in vitro, we used CCK-8 assays and found that DHRS4-AS1 

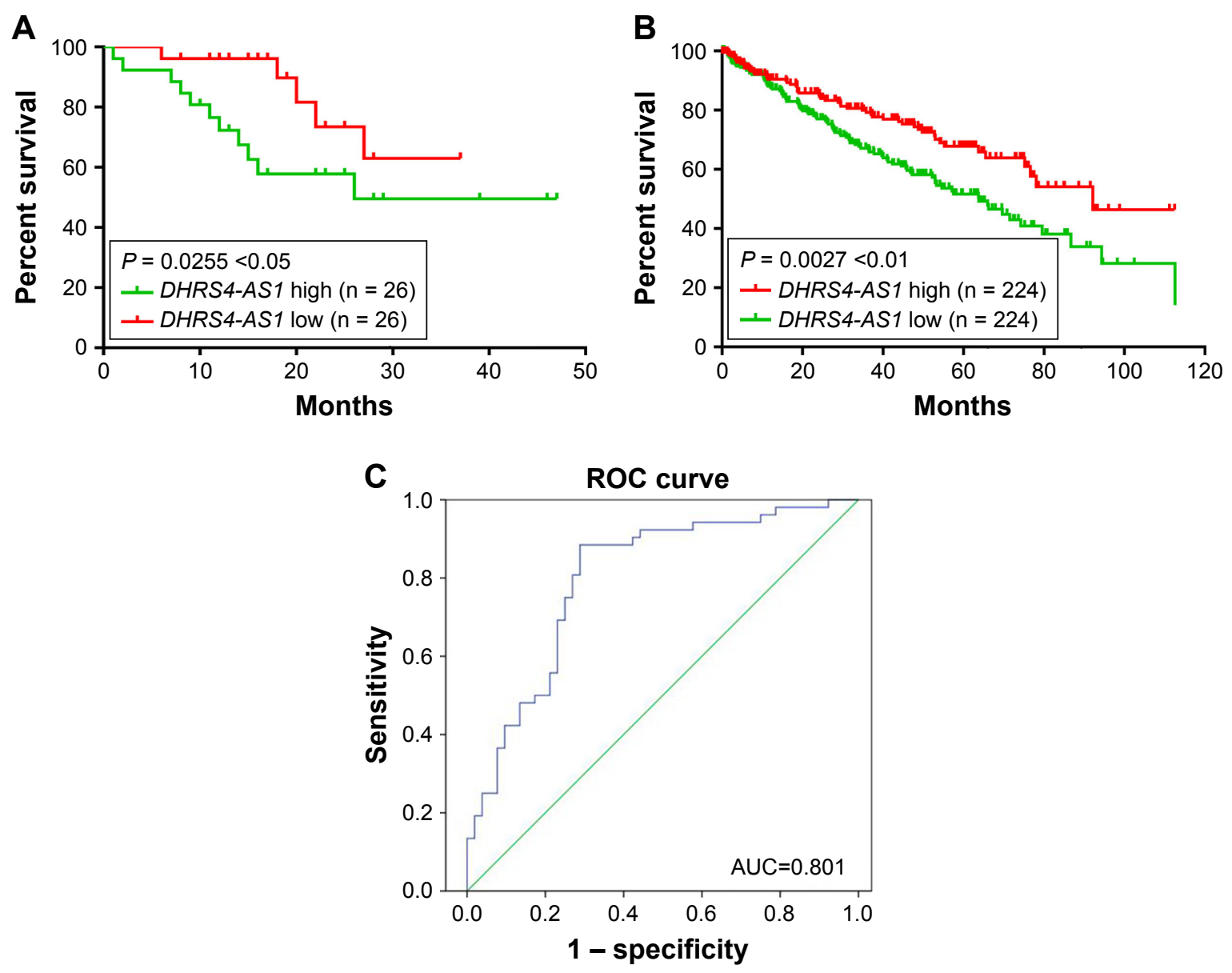

Figure 2 Association between DHRS4-ASI expression and clinicopathological characteristics and survival.

Notes: (A) Kaplan-Meier survival curve based on high and low DHRS4-ASI expression in patients with ccRCC. "DHRS4-ASI low" (n = 26) represents the bottom of the cases, whereas "DHRS4-ASI high" ( $n=26)$ is designated the top of the samples. $(P=0.0255)$. (B) Kaplan-Meier survival curve based on high and low DHRS4-ASI expression in patients with ccRCC (TCGA). "DHRS4-ASI low" $(\mathrm{N}=223)$ represents the bottom of the cases, whereas "DHRS4-ASI high" ( $\mathrm{N}=223)$ is designated the top of the samples $(P=0.0027)$. (C) The ROC analysis revealed that a larger AUC for DHRS4-ASI in ccRCC indicates greater potential as a biomarker $(A U C=0.80 \mathrm{I})$.

Abbreviations: DHRS4-ASI, DHRS4 antisense RNA I; ccRCC, clear cell renal cell carcinoma; TCGA, The Cancer Genome Atlas; ROC, receiver operating characteristic; AUC, area under the curve.

overexpression obviously suppressed tumor cell proliferation compared with the control (Figure 4B). DHRS4-AS1depleted 786-O and 769-P cells formed significantly fewer colonies compared with the control in the colony formation assay (Figure 4C), indicating an important role of DHRS4$A S 1$ in inhibiting the growth of ccRCC cells. To identify the mechanism of DHRS4-AS1 in suppressing cell proliferation, we studied whether DHRS4-AS1 modulates cell cycle distribution by flow cytometry. The cell cycle analysis revealed that, compared with DHRS4-ASI overexpression, the Flag control caused significantly greater accumulation of ccRCC cells in the G0/G1 phase and a reduced number of cells

Table 3 Univariate and multivariate regression analyses of parameters associated with the prognosis of ccRCC patients

\begin{tabular}{|c|c|c|c|c|c|}
\hline \multirow[t]{2}{*}{ Characteristic } & \multirow[t]{2}{*}{ Subset } & \multicolumn{2}{|l|}{ Univariate analysis } & \multicolumn{2}{|c|}{ Multivariate analysis } \\
\hline & & HR (95\% CI) & $P$-value & HR (95\% CI) & P-value \\
\hline Gender & Male/female & $0.956(0.409-2.237)$ & 0.918 & - & - \\
\hline Age (years) & $\leq 60 />60$ & $0.443(0.174-1.126)$ & 0.443 & - & - \\
\hline Tumor stage & $\mathrm{I}+\mathrm{II} / \mathrm{III}+\mathrm{IV}$ & $7.38 \mid(2.94|-| 8.525)$ & $<0.00 I^{*}$ & $2.558(1.589-4.120)$ & $<0.00$ I* \\
\hline Tumor grade & Grade $(I+2) /$ grade $(3+4)$ & $2.373(1.014-5.556)$ & $0.046 *$ & - & - \\
\hline Lymph node metastasis & Yes/no & $6.034(2.503-14.543)$ & $<0.001 *$ & - & - \\
\hline Distant metastasis & Yes/no & $5.444(1.966-15.075)$ & $0.001 *$ & - & - \\
\hline DHRS4-ASI & High/low & $0.407(0.173-0.960)$ & $0.04 *$ & - & - \\
\hline
\end{tabular}

Notes: $* P<0.05$. - indicates no data calculated.

Abbreviations: ccRCC, clear cell renal cell carcinoma; HR, hazard ratio; CI, confidence interval; DHRS4-ASI, DHRS4 antisense RNA I. 
A

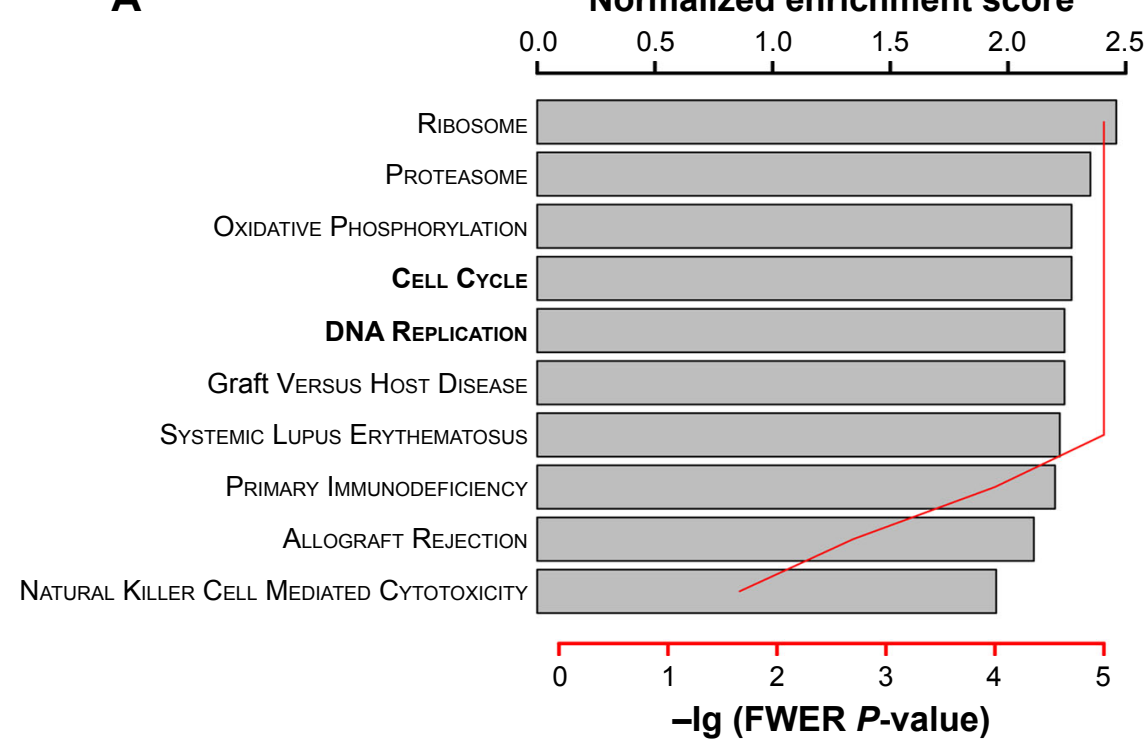

B

KEGG CELL CYCLE

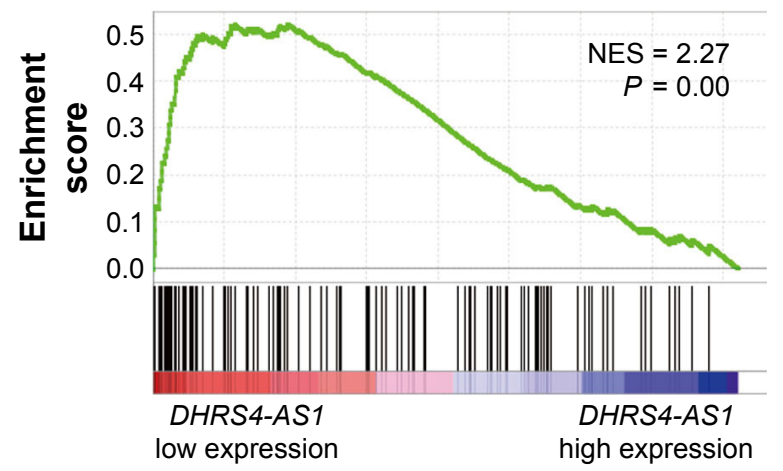

C

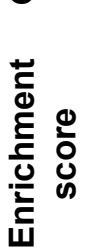

Figure 3 The target gene enrichment of the KEGG pathways of the DHRS4-ASI signature.

Notes: (A) The top 10 KEGG pathways with the highest normalized enrichment scores. GSEA plots presenting the inverse correlation between DHRS4-ASI expression and the negative regulation of cell cycle genes (B) and DNA replication genes in the TCGA KIRC data set (C).

Abbreviations: DHRS4-ASI, DHRS4 antisense RNA I; GSEA, Gene Set Enrichment Analysis; NES, normalized enrichment score; TCGA, The Cancer Genome Atlas.

in the $\mathrm{S}$ phase (Figure 5A). These results suggest that the upregulation of DHRS4-AS1 suppresses the proliferation of ccRCC cells possibly by inhibiting cell cycle progression.

We also studied whether DHRS4-AS1 overexpression inhibits the proliferation of ccRCC cells by influencing apoptosis. Flow cytometry revealed that lentiviral transfectioninduced DHRS4-AS1 overexpression induced apoptosis in ccRCC cells (Figure 5B), especially early apoptosis, indicating that DHRS4-ASI has a proapoptotic effect.

\section{In vitro overexpression of DHRS4-ASI suppresses ccRCC cell line migration and invasion}

Considering that lower DHRS4-AS1 expression is significantly correlated with lymph node metastasis in ccRCC, we reasoned that DHRS4-AS1 plays a role in inhibiting ccRCC cell motility. As shown in Figure 6A, DHRS4-AS1 overexpression significantly inhibited 786-O and 769-P cells' motility as measured by a wound-healing assay (Figure 6B). The migration alternation was further confirmed by transwell assays (Figure 6C and D). DHRS4-AS1 overexpression suppressed 786-O and 769-P cell migration. Therefore, DHRS4-AS1 has a critical role in the invasion and metastasis of ccRCC.

\section{DHRS4-ASI controls the expression of epithelial-mesenchymal transition (EMT) associated genes in ccRCC cells}

To further explore the mechanism by which DHRS4-AS1 regulates cell migration and invasion, we examined protein levels of metastasis-associated and EMT-associated genes in ccRCC cells with overexpression of DHRS4-AS1 expression. We found that, DHRS4-AS1 overexpression significantly decreased the expression of mesenchymal marker 
A

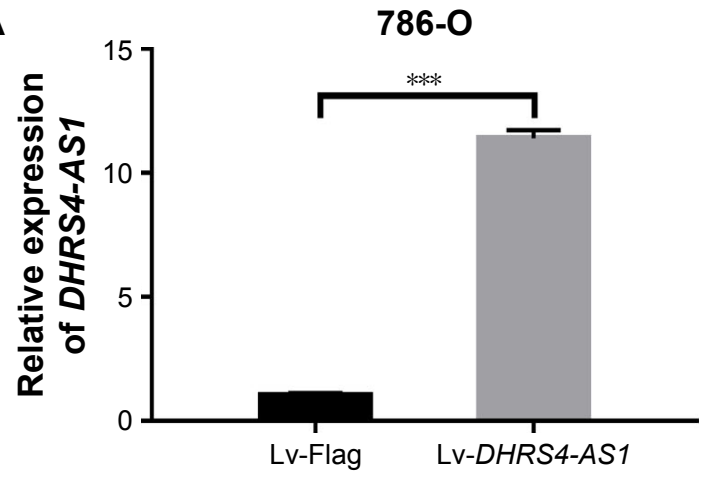

B

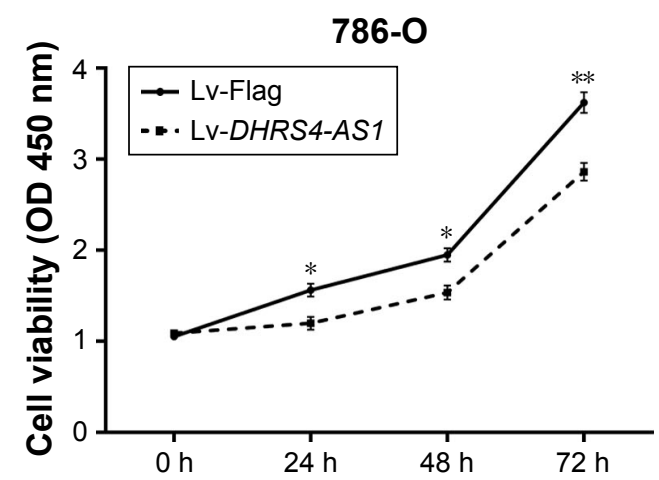

C
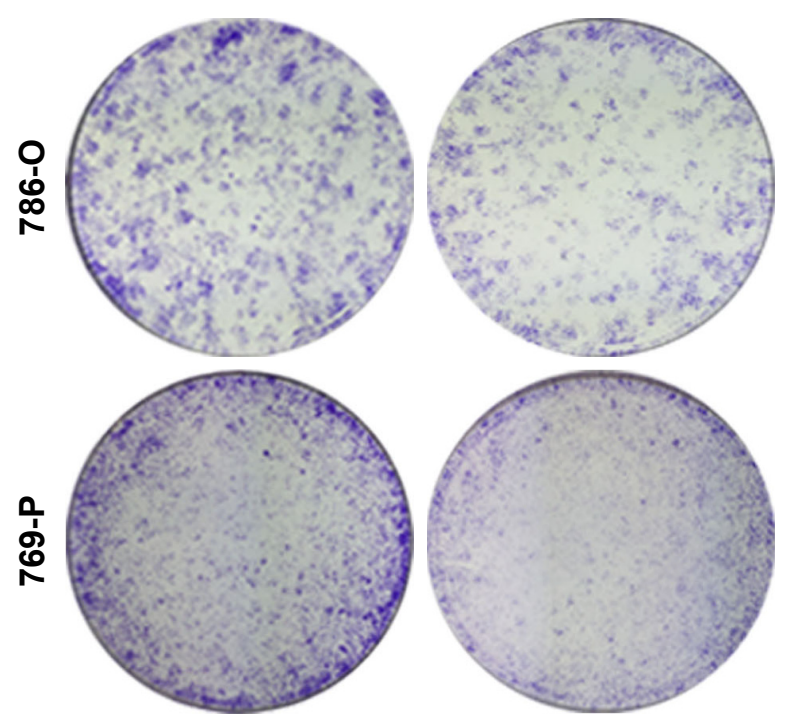

Lv-Flag

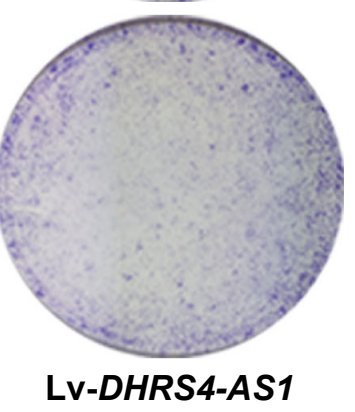

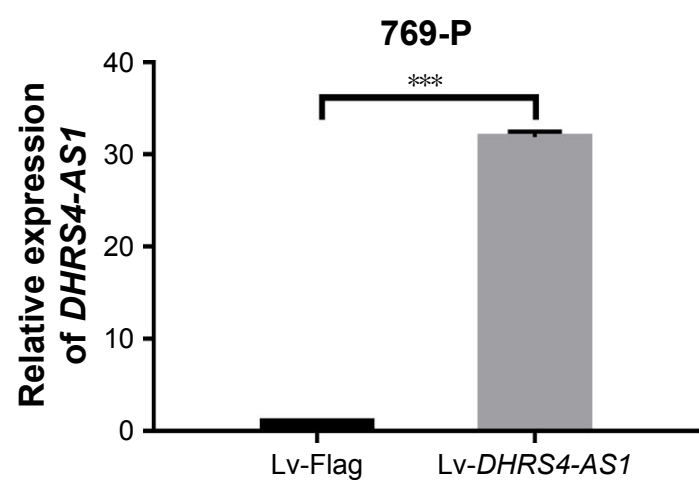
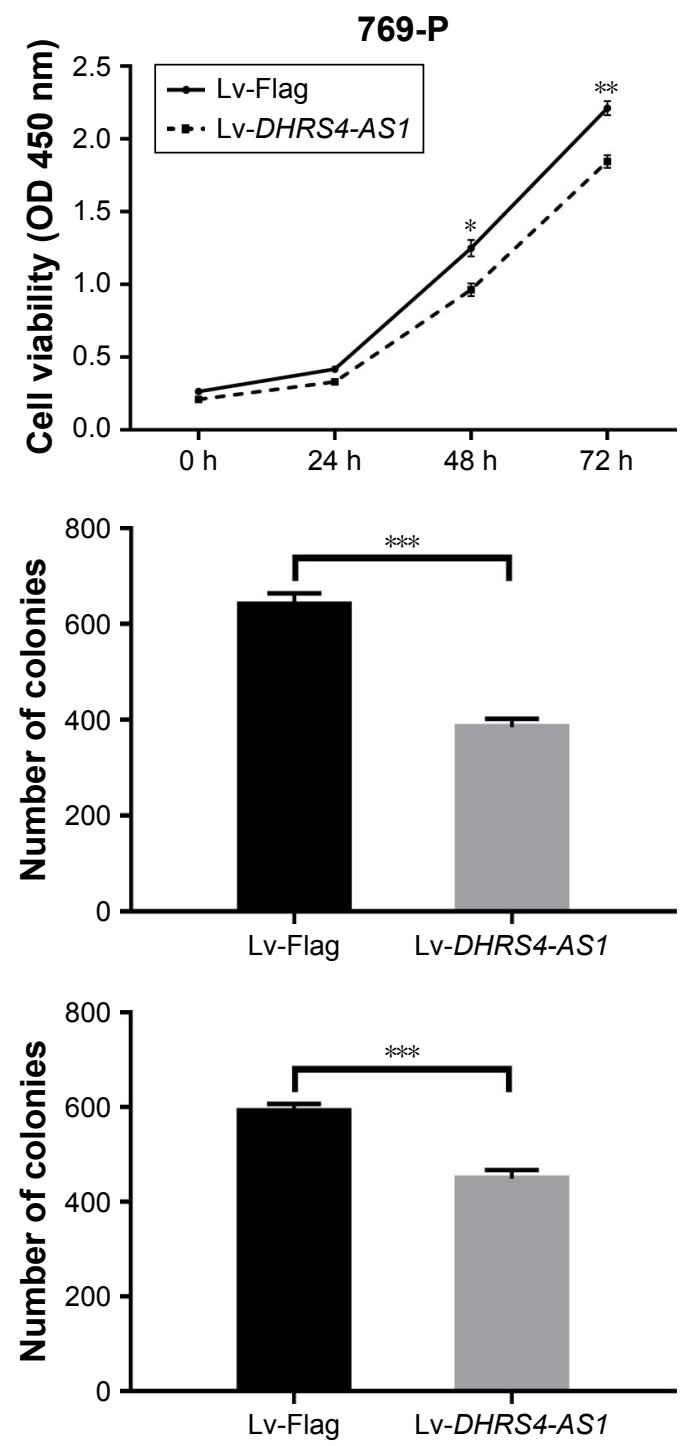

Figure 4 Exogenetic DHRS4-ASI expression suppresses ccRCC cell proliferation and colony formation in vitro.

Notes: (A) Real-time PCR analysis of DHRS4-ASI in 786-O and 769-P cells after exogenetic expression. (B) Ectopic DHRS4-ASI overexpression inhibits proliferation

(C) and decreases colony formation in 786-O and 769-P cell lines. For all statistical analyses: $* P<0.05 ; * * P<0.01$; ***P<0.00I. The data are presented as the mean \pm SD. Experiments were performed three times.

Abbreviations: DHRS4-ASI, DHRS4 antisense RNA I; ccRCC, clear cell renal cell carcinoma; Lv, lentivirus.

genes including vimentin and $\mathrm{N}$-cadherin and increased the expression of epithelial marker genes including E-cadherin, suggesting that overexpression of DHRS4-AS1 could inhibit the EMT process (Figure 7A and B).

\section{Discussion}

ccRCC, which is characterized by its clear cell morphology phenotypic feature, has been related to lipid and glycogen accumulation. ${ }^{27}$ Moreover, ccRCC is one of the most 

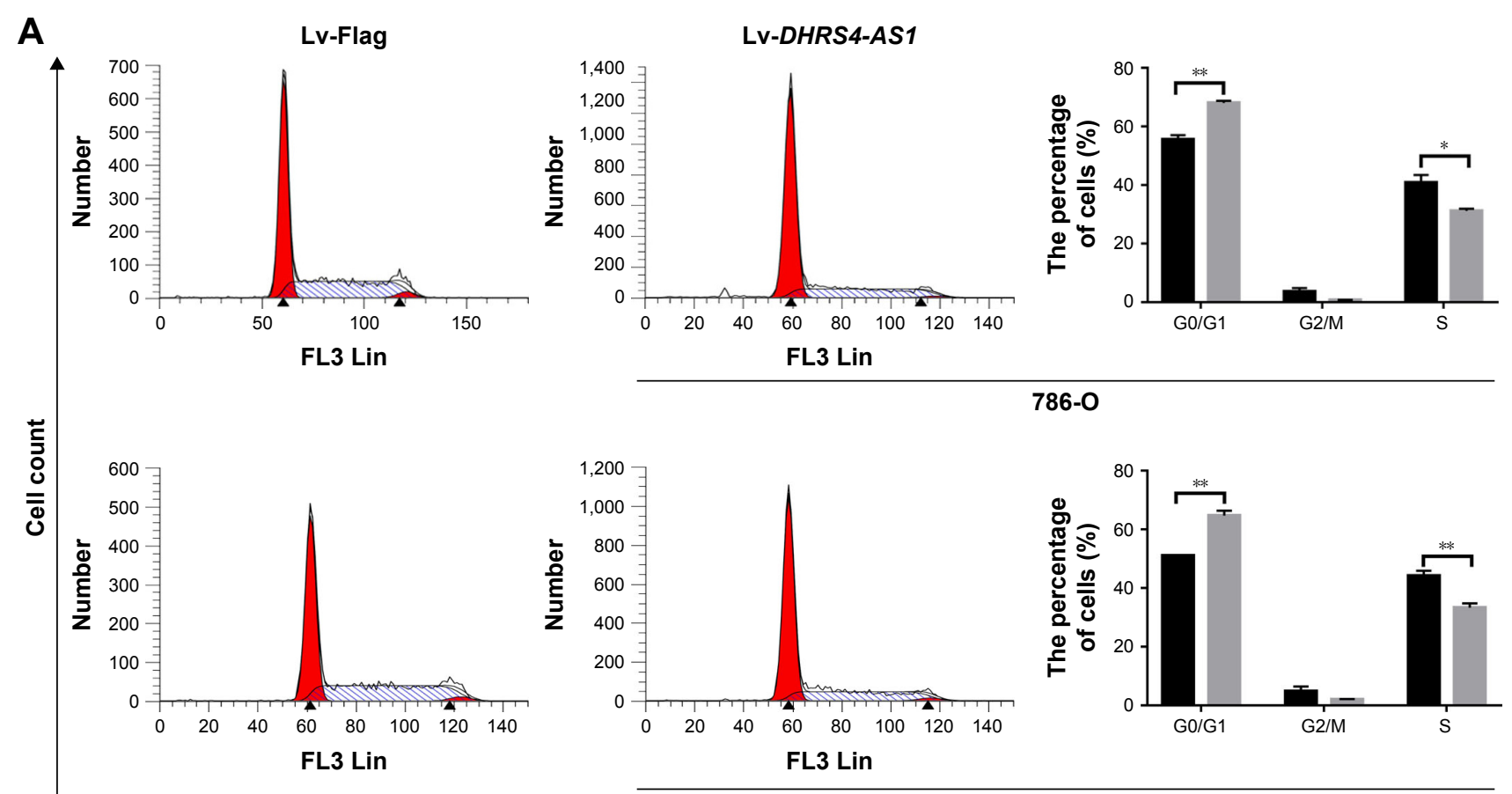

769-P
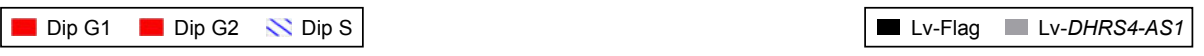

DNA content

B
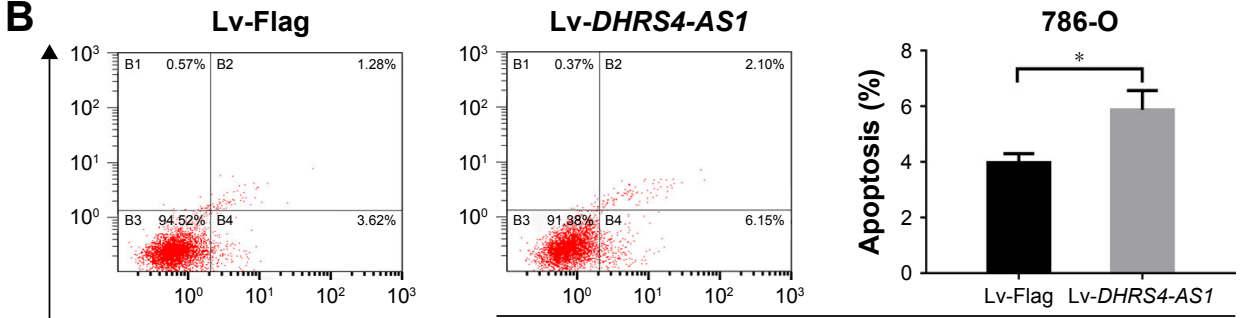

$\stackrel{\text { 电 }}{\leqslant}$

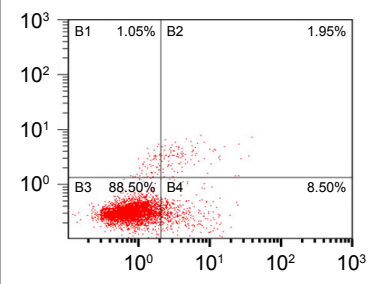

769-P
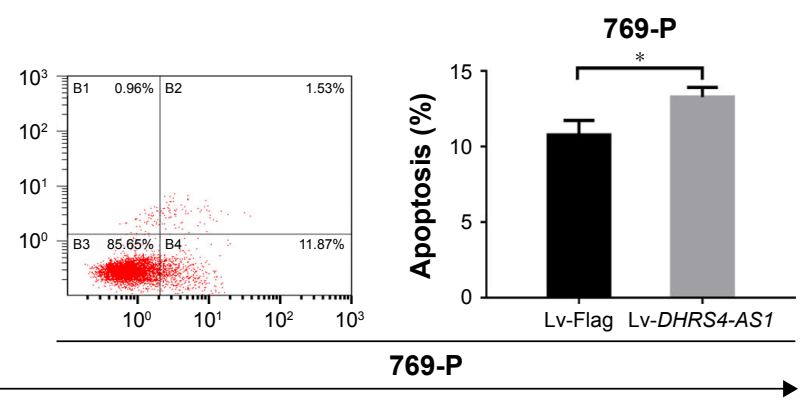

PE

Figure 5 Exogenetic DHRS4-ASI expression induced apoptosis and cell cycle arrest in ccRCC cells.

Notes: (A) Flow cytometry was used to assess the cell cycle distribution of 786-O and 769-P cells transfected for $24 \mathrm{~h}$ and stained with PI. Representative and quantitative results are shown. (B) Flow cytometry was used to assess the percentage of apoptotic 786-O and 769-P cells after transfection for $48 \mathrm{~h}$. Representative scatter plots and quantitative results are shown. $\mathrm{n}=3$; $* \mathrm{P}<0.05$; $* * \mathrm{P}<0.0 \mathrm{I}$.

Abbreviations: DHRS4-ASI, DHRS4 antisense RNA I; ccRCC, clear cell renal cell carcinoma; Lv, lentivirus; PI, propidium iodide; PE, phycoerythrin; Lin, linea.

therapy-resistant cancers and responds very poorly or not at all to radiotherapy, chemotherapy and hormonal therapy. ${ }^{28}$ Most ccRCC patients are diagnosed at a later stage, leading to ineffective treatments. ${ }^{29}$ These facts emphasize the importance of developing novel prognostic biomarkers and therapeutic targets for ccRCC to improve the outcomes of patients.

Recent studies have shown that lncRNAs, defined as RNA transcripts over 200 nucleotides, are aberrantly expressed in ccRCC tissues and play pivotal roles in the regulation of 


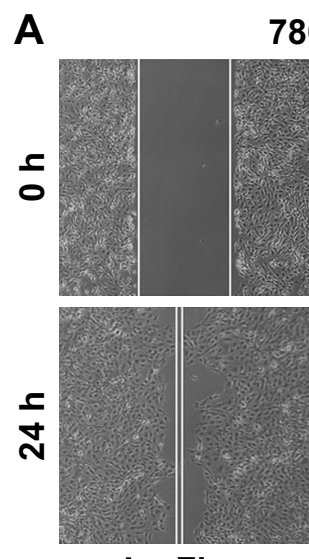

Lv-Flag
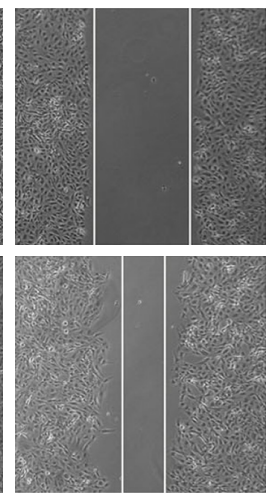

Lv-DHRS4-AS1
B

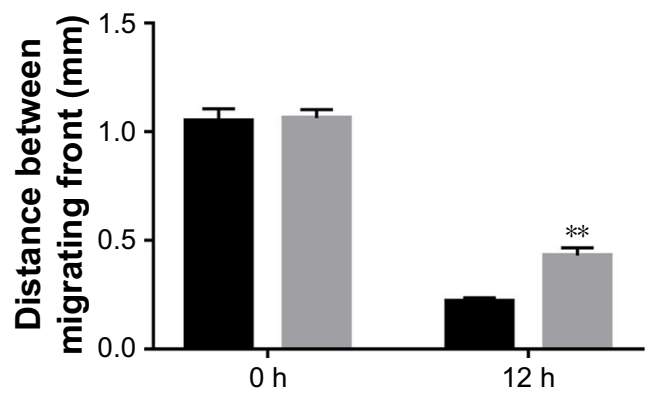

769-P
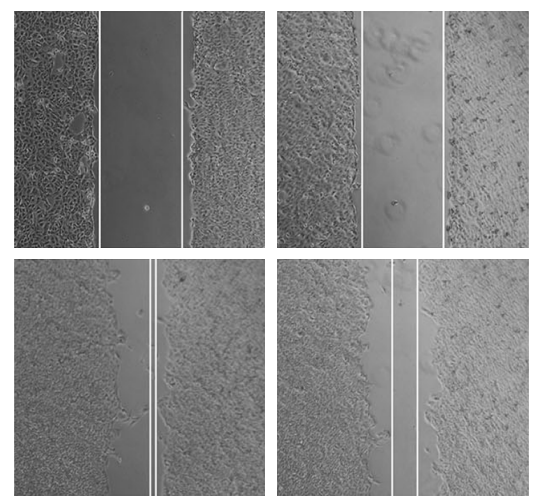

Lv-Flag

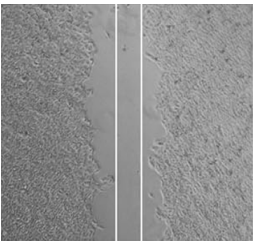

Lv-DHRS4-AS1

769-P

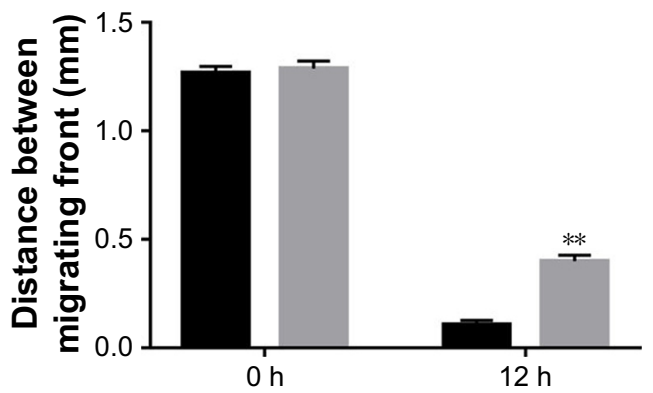

Lv-Flag Lv-DHRS4-AS1
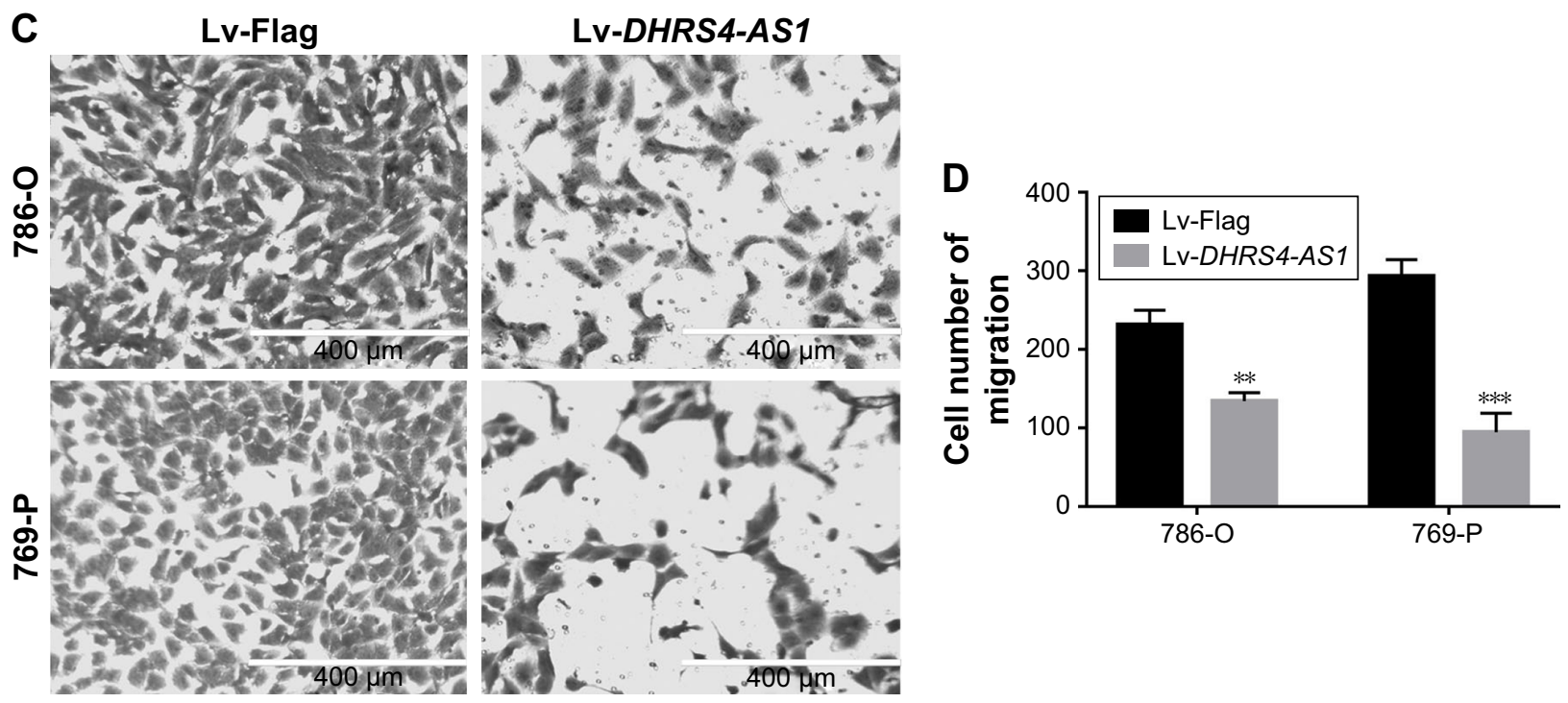

Figure 6 Exogenetic DHRS4-ASI expression suppresses ccRCC cell migration.

Notes: (A and B) In vitro wound-healing analysis in negative control, Flag or DHRS4-ASI cells. Representative photographs and quantitative results $24 \mathrm{~h}$ after generating the wound are presented. (C and D) The migration rate was also detected by transwell assay in negative control, Flag or DHRS4-ASI cells. Representative photographs and quantitative results are presented. For all statistical analyses: $* * P<0.01$; $* * * P<0.001$. The data are presented as the mean \pm SD. Experiments were performed three times.

Abbreviations: DHRS4-ASI, DHRS4 antisense RNA I; ccRCC, clear cell renal cell carcinoma; Lv, lentivirus.

ccRCC. ${ }^{30,31}$ They are associated with the pathogenesis, progression and metastasis of ccRCC. They are also involved in the tumorigenic process of ccRCC, including cell cycle control, cell proliferation, cell apoptosis, invasion and metastasis. ${ }^{32-36}$ For example, MALAT1 was found to be upregulated in ccRCC tissues and to be associated with poor prognosis. ${ }^{37}$ Conversely, IncRNA CADM1-AS1 functions as a tumor suppressor in ccRCC. ${ }^{38}$ Many studies have 

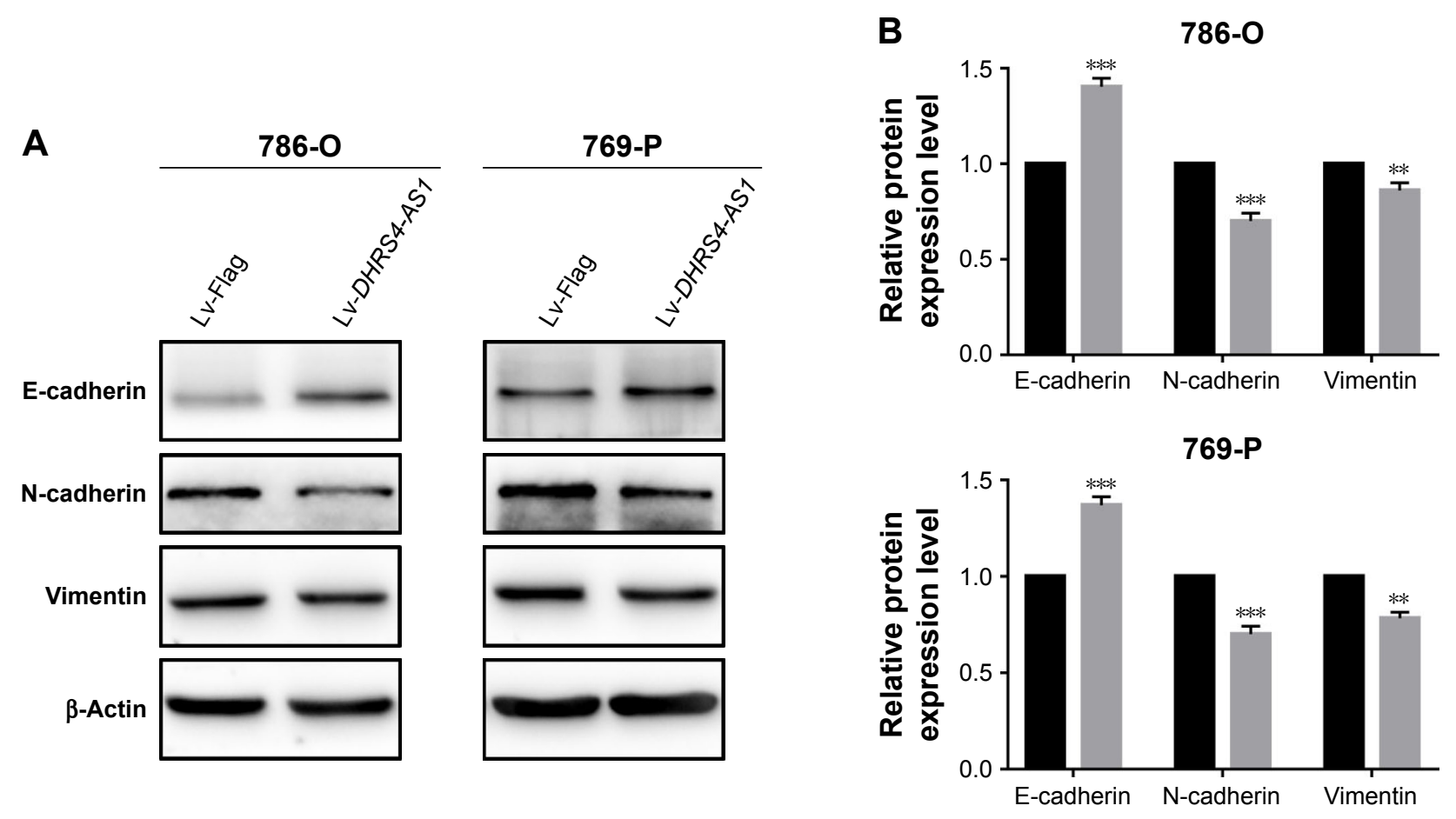

Lv-Flag Lv-DHRS4-AS1

Figure 7 DHRS4-ASI controls the expression of EMT-associated genes in ccRCC cells.

Notes: $(\mathbf{A}$ and $\mathbf{B})$ The expression levels of E-cadherin, $\mathrm{N}$-cadherin and Vimentin in 786-O and 769-P cells were measured by using Western blot analysis. **P $<0.01$; $* * * P<0.001$. The data are presented as the mean \pm SD. Experiments were performed three times.

Abbreviations: DHRS4-ASI, DHRS4 antisense RNA I; ccRCC, clear cell renal cell carcinoma; EMT, epithelial-mesenchymal transition; Lv, lentivirus.

demonstrated that abnormal expression levels of specific IncRNAs in ccRCC could serve as independent diagnostic and prognostic biomarkers. ${ }^{39,40}$ Recently, several studies have found that lncRNAs play a critical role in ccRCC tumorigenesis. $^{41}$

The DHRS4 gene cluster is localized on 14q11.2. The DHRS4 gene codes for short-chain dehydrogenase/reductases that participate in retinoic acid and steroid pathways and are associated with cell proliferation, differentiation and tumorigenesis. $^{42} D H R S 4-A S 1$, which is a head-to-head natural antisense transcript (NAT) of DHRS4, silences all three genes of the DHRS4 gene cluster (DHRS4, DHRS4L2 and $D H R S 4 L 1$ ) through the recruitment of epigenetic modifiers. ${ }^{22,23}$ Whether DHRS4-AS1 affects tumor proliferation, differentiation, and cell cycle progression by manipulating the expression of DHRS4, thereby affecting the stability of retinoic acid, remains unknown. Little is known about the significance of DHRS4-AS1 expression in carcinomas.

In the current study, we found that the expression of DHRS4-AS1 was statistically lower in renal cancer cell lines and in ccRCC tissues than in the normal human proximal tubule epithelial cell line HK-2 and adjacent non-tumor tissues. Moreover, a lower DHRS4-AS1 expression level is significantly associated with lymph node metastasis, a high TNM stage, advanced pathological grade and a poor overall survival. These data suggest that low DHRS4-AS1 expression may serve as a novel indicator of poor prognosis in ccRCC and may be a potential therapeutic target for diagnosis and gene therapy. Then we tried to guess the reason which resulted in the downregulation of DHRS4-AS1, as methylation is the most common cause of gene silencing, so we browsed the UCSC data base and found there is methylation island in the promoter region of DHRS4-AS1 (Figure S1A). ${ }^{43,44}$ Based on this prediction, we treated the cells with 5-azacytidine and DMSO as a control. After $72 \mathrm{~h}$, the cells were harvested, then $\mathrm{qRT}$-PCR was executed to estimate the expression of DHRS4-AS1, as shown in Figure S1B the expression of DHRS4-AS1 was significantly downregulated in 5-azacytidine treatment group compare with the control group. These results suggest that the methylation of DHRS4-AS1 promoter may be one of the reasons for its low expression in ccRCC.

One classic approach to exploring the putative functions of lncRNAs is through a "guilt-by-association" method. ${ }^{45}$ This method associates lncRNAs with biological processes based on common expression patterns and identifies lncRNAs 
that are associated with specific cellular processes. ${ }^{46,47}$ To explore the function of DHRS4-AS1 in ccRCC, our GSEA data indicated that DHRS4-AS1 overexpression was negatively correlated with cell cycle genes and DNA replication genes (Figure 3B and C). Cell cycle regulation is frequently abnormal in most common malignancies, resulting in aberrant cell proliferation. ${ }^{48,49}$ Here, DHRS4-AS1 significantly promoted cell arrest in the G1 phase (Figure 5A), suppressing the proliferation of ccRCC cells by inhibiting cell cycle progression. Meanwhile, DHRS4-AS1 upregulation significantly suppressed the proliferation of ccRCC cells as shown by the CCK-8 assay. We then evaluated the effect of DHRS4-AS1 on 786-O and 769-P cells relative to apoptosis. DHRS4-AS1 upregulation increased the apoptosis of ccRCC cells, especially early apoptosis. Abundant evidence shows that retinoic acid inhibits cell cycle progression and induces the process of apoptosis in a variety of human cancer cells via various signaling pathways. ${ }^{42}$ A potential explanation is that DHRS4-AS1 can affect the stability of retinoic acid and subsequently play a role in inhibiting tumor growth and promoting apoptosis. The above data suggest that DHRS4-ASI may inhibit tumor growth by inhibiting the progression of the cell cycle, maintaining the tumor at a lower clinical grade and pathological stage (Table 2). In conclusion, DHRS4-AS1 may be an important factor that affects tumor cell proliferation, suggesting that DHRS4-AS1 is an important factor in ccRCC prognosis.

Invasion and metastasis are two of the most important hallmarks of malignant tumors and are directly correlated with mortality in human cancers. ${ }^{50}$ In this study, low DHRS4$A S 1$ expression was significantly correlated with lymph node metastasis in ccRCC (Table 2). To further explore the function of DHRS4-AS1 in the ccRCC cell process, our in vitro experiments revealed that DHRS4-AS1 overexpression significantly suppressed the invasion and migration capabilities of ccRCC cells by transwell assay. Therefore, our study suggests that DHRS4-AS1 is an important regulator in controlling ccRCC cell metastasis. However, further studies are needed to identify the underlying molecular mechanisms of DHRS4-AS1 in the metastasis of ccRCC.

\section{Conclusion}

Collectively, we first identified the critical role of DHRS4$A S 1$ in ccRCC. Our research found the following: 1) DHRS4$A S 1$ was downregulated in ccRCC tissues and cell lines; 2) DHRS4-AS1 expression was associated with lymph node metastasis, a high TNM stage and postoperative survival in renal cell cancer patients and 3) DHRS4-AS1 upregulation significantly suppressed ccRCC cell proliferation, arrested the cell cycle, facilitated apoptosis and inhibited ccRCC cell migration. These data show that the lncRNA DHRS4$A S 1$ exhibits antitumor activity and that DHRS4-AS1 may be a novel target in ccRCC diagnostic and therapeutic strategies.

\section{Acknowledgments}

This study was funded by the National Natural Science Foundation of China (grant numbers 81171996 and 81272289 to Cheng Zhang), the Wujieping Medical Foundation (grant number 320.6750.13252 to Cheng Zhang), and the First Affiliated Hospital of Harbin Medical University Science Foundation (grant number 2015B011). Changlin Wang, Gang Wang and Zijian Zhang are considered to be co-first authors.

\section{Disclosure}

The authors report no conflicts of interest in this work.

\section{References}

1. Turajlic S, Larkin J, Swanton C. SnapShot: renal cell carcinoma. Cell. 2015;163(6):1556-1556.e1.

2. Cheville JC, Lohse CM, Zincke H, Weaver AL, Blute ML. Comparisons of outcome and prognostic features among histologic subtypes of renal cell carcinoma. Am J Surg Pathol. 2003;27(5):612-624.

3. Janzen NK, Kim HL, Figlin RA, Belldegrun AS. Surveillance after radical or partial nephrectomy for localized renal cell carcinoma and management of recurrent disease. Urol Clin North Am. 2003;30(4): $843-852$.

4. Bukowski RM. Metastatic clear cell carcinoma of the kidney: therapeutic role of bevacizumab. Cancer Manag Res. 2010;2:83-96.

5. Gupta K, Miller JD, Li JZ, Russell MW, Charbonneau C. Epidemiologic and socioeconomic burden of metastatic renal cell carcinoma (mRCC): a literature review. Cancer Treat Rev. 2008;34(3):193-205.

6. Wahlestedt $\mathrm{C}$. Targeting long non-coding RNA to therapeutically upregulate gene expression. Nat Rev Drug Discov. 2013;12(6):433-446.

7. Ponting CP, Oliver PL, Reik W. Evolution and functions of long noncoding RNAs. Cell. 2009;136(4):629-641.

8. Eissmann M, Gutschner T, Hammerle M, et al. Loss of the abundant nuclear non-coding RNA MALAT1 is compatible with life and development. RNA Biol. 2012;9(8):1076-1087.

9. Rossi MN, Antonangeli F. LncRNAs: new players in apoptosis control Int J Cell Biol. 2014;2014:473857.

10. DeOcesano-Pereira C, Amaral MS, Parreira KS, et al. Long non-coding RNA INXS is a critical mediator of BCL-XS induced apoptosis. Nucleic Acids Res. 2014;42(13):8343-8355.

11. Pandey GK, Mitra S, Subhash S, et al. The risk-associated long noncoding RNA NBAT-1 controls neuroblastoma progression by regulating cell proliferation and neuronal differentiation. Cancer Cell. 2014; 26(5):722-737.

12. Prensner JR, Chinnaiyan AM. The emergence of lncRNAs in cancer biology. Cancer Discov. 2011;1(5):391-407.

13. Fang Y, Wang J, Wu F, Song Y, Zhao S, Zhang Q. Long non-coding RNA HOXA-AS2 promotes proliferation and invasion of breast cancer by acting as a miR-520c-3p sponge. Oncotarget. 2017;8(28): 46090-46103.

14. Wei W, Liu Y, Lu Y, Yang B, Tang L. LncRNA XIST promotes pancreatic cancer proliferation through miR-133a/EGFR. J Cell Biochem. 2017;118(10):3349-3358 
15. Li N, Wang Y, Liu X, et al. Identification of circulating long noncoding RNA HOTAIR as a novel biomarker for diagnosis and monitoring of non-small cell lung cancer. Technol Cancer Res Treat. 2017: 1533034617723754

16. Quagliata L, Matter MS, Piscuoglio S, et al. Long noncoding RNA HOTTIP/HOXA13 expression is associated with disease progression and predicts outcome in hepatocellular carcinoma patients. Hepatology. 2014;59(3):911-923.

17. Handa H, Kuroda Y, Kimura K, et al. Long non-coding RNA MALAT1 is an inducible stress response gene associated with extramedullary spread and poor prognosis of multiple myeloma. Br J Haematol. 2017; 179(3):449-460.

18. Cao Y, Shi H, Ren F, Jia Y, Zhang R. Long non-coding RNA CCAT1 promotes metastasis and poor prognosis in epithelial ovarian cancer. Exp Cell Res. 2017;359(1):185-194.

19. Ellinger J, Alam J, Rothenburg J, et al. The long non-coding RNA lncZNF180-2 is a prognostic biomarker in patients with clear cell renal cell carcinoma. Am J Cancer Res. 2015;5(9):2799-2807.

20. Liu Z, Yan HY, Xia SY, Zhang C, Xiu YC. Downregulation of long non-coding RNA TRIM52-AS1 functions as a tumor suppressor in renal cell carcinoma. Mol Med Rep. 2016;13(4):3206-3212.

21. Su H, Sun T, Wang H, et al. Decreased TCL6 expression is associated with poor prognosis in patients with clear cell renal cell carcinoma. Oncotarget. 2017;8(4):5789-5799.

22. Yang Y, Su Z, Song X, et al. Enhancer RNA-driven looping enhances the transcription of the long noncoding RNA DHRS4-AS1, a controller of the DHRS4 gene cluster. Sci Rep. 2016;6:20961.

23. Li Q, Su Z, Xu X, et al. AS1DHRS4, a head-to-head natural antisense transcript, silences the DHRS4 gene cluster in cis and trans. Proc Natl Acad Sci U S A. 2012;109(35):14110-14115.

24. Cline MS, Craft B, Swatloski T, et al. Exploring TCGA pan-cancer data at the UCSC cancer genomics browser. Sci Rep. 2013;3:2652.

25. Subramanian A, Tamayo P, Mootha VK, et al. Gene set enrichment analysis: a knowledge-based approach for interpreting genome-wide expression profiles. Proc Natl Acad Sci U S A. 2005;102(43):15545-15550.

26. Du Z, Li L, Huang X, et al. The epigenetic modifier CHD5 functions as a novel tumor suppressor for renal cell carcinoma and is predominantly inactivated by promoter $\mathrm{CpG}$ methylation. Oncotarget. 2016; 7(16):21618-21630.

27. Rezende RB, Drachenberg CB, Kumar D, et al. Differential diagnosis between monomorphic clear cell adenocarcinoma of salivary glands and renal (clear) cell carcinoma. Am J Surg Pathol. 1999;23(12): 1532-1538.

28. Zaravinos A, Pieri M, Mourmouras N, et al. Altered metabolic pathways in clear cell renal cell carcinoma: a meta-analysis and validation study focused on the deregulated genes and their associated networks. Oncoscience. 2014;1(2):117-131.

29. Escudier B, Porta C, Schmidinger M, et al; ESMO Guidelines Working Group. Renal cell carcinoma: ESMO clinical practice guidelines for diagnosis, treatment and follow-up. Ann Oncol. 2016;25(suppl 3): iii49-iii56.

30. Deng M, Blondeau JJ, Schmidt D, Perner S, Muller SC, Ellinger J. Identification of novel differentially expressed lncRNA and mRNA transcripts in clear cell renal cell carcinoma by expression profiling. Genom Data. 2015;5:173-175.

31. Blondeau JJ, Deng M, Syring I, et al. Identification of novel long non-coding RNAs in clear cell renal cell carcinoma. Clin Epigenetics. 2015;7:10.
32. Zhang HM, Yang FQ, Yan Y, Che JP, Zheng JH. High expression of long non-coding RNA SPRY4-IT1 predicts poor prognosis of clear cell renal cell carcinoma. Int J Clin Exp Pathol. 2014;7(9):5801-5809.

33. Wu Y, Liu J, Zheng Y, You L, Kuang D, Liu T. Suppressed expression of long non-coding RNA HOTAIR inhibits proliferation and tumourigenicity of renal carcinoma cells. Tumour Biol. 2014;35(12): 11887-11894.

34. Xiong J, Liu Y, Jiang L, Zeng Y, Tang W. High expression of long noncoding RNA lncRNA-ATB is correlated with metastases and promotes cell migration and invasion in renal cell carcinoma. Jpn J Clin Oncol. 2016;46(4):378-384.

35. Zhang M, Lu W, Huang Y, et al. Downregulation of the long noncoding RNA TUG1 inhibits the proliferation, migration, invasion and promotes apoptosis of renal cell carcinoma. J Mol Histol. 2016;47(4):421-428.

36. Posa I, Carvalho S, Tavares J, Grosso AR. A pan-cancer analysis of MYC-PVT1 reveals CNV-unmediated deregulation and poor prognosis in renal carcinoma. Oncotarget. 2016;7(30):47033-47041.

37. Hirata H, Hinoda Y, Shahryari V, et al. Long noncoding RNA MALAT1 promotes aggressive renal cell carcinoma through Ezh2 and interacts with miR-205. Cancer Res. 2015;75(7):1322-1331.

38. Yao J, Chen Y, Wang Y, et al. Decreased expression of a novel lncRNA CADM1-AS1 is associated with poor prognosis in patients with clear cell renal cell carcinomas. Int J Clin Exp Pathol. 2014;7(6):2758-2767.

39. Song S, Wu Z, Wang C, et al. RCCRT1 is correlated with prognosis and promotes cell migration and invasion in renal cell carcinoma. Urology. 2014;84(3):730.e1-e7.

40. Wang L, Cai Y, Zhao X, et al. Down-regulated long non-coding RNA H19 inhibits carcinogenesis of renal cell carcinoma. Neoplasma. 2015; 62(3):412-418.

41. Liu Y, Qian J, Li X, et al. Long noncoding RNA BX357664 regulates cell proliferation and epithelial-to-mesenchymal transition via inhibition of TGF-beta1/p38/HSP27 signaling in renal cell carcinoma. Oncotarget. 2016;7(49):81410-81422.

42. Tang XH, Gudas LJ. Retinoids, retinoic acid receptors, and cancer. Annu Rev Pathol. 2011;6:345-364.

43. Guo W, Liu S, Dong Z, et al. Aberrant methylation-mediated silencing of lncRNA CTC-276P9.1 is associated with malignant progression of esophageal squamous cell carcinoma. Clin Exp Metastasis. 2018;35(1-2): 53-68.

44. Rokavec M, Horst D, Hermeking H. Cellular model of colon cancer progression reveals signatures of mRNAs, miRNA, lncRNAs, and epigenetic modifications associated with metastasis. Cancer Res. 2017;77(8):1854-1867.

45. Cabili MN, Trapnell C, Goff L, et al. Integrative annotation of human large intergenic noncoding RNAs reveals global properties and specific subclasses. Genes Dev. 2011;25(18):1915-1927.

46. Liao Q, Liu C, Yuan X, et al. Large-scale prediction of long non-coding RNA functions in a coding-non-coding gene co-expression network. Nucleic Acids Res. 2011;39(9):3864-3878.

47. Guo X, Gao L, Liao Q, et al. Long non-coding RNAs function annotation: a global prediction method based on bi-colored networks. Nucleic Acids Res. 2013;41(2):e35.

48. Molinari M. Cell cycle checkpoints and their inactivation in human cancer. Cell Prolif. 2000;33(5):261-274

49. Evan GI, Vousden KH. Proliferation, cell cycle and apoptosis in cancer. Nature. 2001;411(6835):342-348.

50. Thiery JP. Epithelial-mesenchymal transitions in tumour progression. Nat Rev Cancer. 2002;2(6):442-454. 


\section{Supplementary materials}

Table SI Associations of DHRS4-ASI expression with clinicopathological factors

\begin{tabular}{|c|c|c|c|c|c|}
\hline \multirow[t]{2}{*}{ Characteristic } & \multirow{2}{*}{$\begin{array}{l}\text { Number of } \\
\text { patients, } n \text { (\%) }\end{array}$} & \multicolumn{2}{|l|}{ DHRS4-ASI } & \multirow[t]{2}{*}{$\chi^{2}$} & \multirow[t]{2}{*}{$P$-value } \\
\hline & & $\begin{array}{l}\text { High } \\
\text { expression (\%) }\end{array}$ & $\begin{array}{l}\text { Low } \\
\text { expression (\%) }\end{array}$ & & \\
\hline Gender & $446(100)$ & 223 & 223 & 0.039 & 0.844 \\
\hline Male & $286(64.13)$ & I 44 (64.57) & $142(63.68)$ & & \\
\hline Female & $160(35.87)$ & $79(35.43)$ & 81 (36.32) & & \\
\hline \multicolumn{2}{|l|}{ Age (years) } & & & 2.894 & 0.089 \\
\hline$\leq 60$ & $218(48.88)$ & $100(44.84)$ & II 8 (52.9I) & & \\
\hline$>60$ & $228(51.12)$ & $123(55.16)$ & 105 (47.09) & & \\
\hline \multicolumn{2}{|l|}{ TNM stage } & & & $|2.23|$ & $0.00 I^{*}$ \\
\hline I-II & $275(61.66)$ & $156(69.96)$ & $119(53.36)$ & & \\
\hline III-IV & I7I (38.34) & $67(30.04)$ & $104(46.6)$ & & \\
\hline \multicolumn{2}{|c|}{ Lymph node metastasis } & & & 0.153 & 0.696 \\
\hline No & $218(48.88)$ & $120(93.75)$ & $98(92.45)$ & & \\
\hline Yes & $16(3.59)$ & $8(6.25)$ & $8(7.55)$ & & \\
\hline \multicolumn{2}{|l|}{ Grade } & & & 6.023 & $0.014^{*}$ \\
\hline GI-G2 & 199 (44.62) & $113(50.90)$ & $86(39.27)$ & & \\
\hline G3-G4 & $242(54.26)$ & $109(49.09)$ & $133(60.73)$ & & \\
\hline
\end{tabular}

Notes: $* p<0.05$. - indicates no data calculated.

Abbreviations: DHRS4-ASI, DHRS4 antisense RNA I; TNM, tumour node metastasis.

Table S2 Univariate and multivariate regression analyses of parameters associated with the prognosis of ccRCC patients

\begin{tabular}{|c|c|c|c|c|c|}
\hline \multirow[t]{2}{*}{ Characteristics } & \multirow[t]{2}{*}{ Subset } & \multirow{2}{*}{$\frac{\text { Univariate analysis }}{\mathrm{HR}(95 \% \mathrm{Cl})}$} & \multirow[t]{2}{*}{$P$-value } & \multirow{2}{*}{$\frac{\text { Multivariate analysis }}{\text { HR }(95 \% \mathrm{Cl})}$} & \multirow[t]{2}{*}{$P$-value } \\
\hline & & & & & \\
\hline Gender & Male/female & $0.985(0.730-1.330)$ & 0.922 & - & - \\
\hline Age (years) & $\leq 60 />60$ & $0.818(0.633-1.059)$ & 0.13 & - & - \\
\hline Tumor stage & $\mathrm{I}+\mathrm{II} / \mathrm{III}+\mathrm{IV}$ & $3.628(2.592-5.077)$ & $<0.00 I^{*}$ & $2.558(1.589-4.120)$ & $<0.00 I^{*}$ \\
\hline Tumor grade & Grade $(I+2) /$ grade $(3+4)$ & $2.490(1.700-3.648)$ & $<0.00 I^{*}$ & $2.052(1.193-3.530)$ & $0.009 *$ \\
\hline Lymph node metastasis & Yes/no & $2.046(1.021-4.100)$ & $0.043^{*}$ & - & - \\
\hline DHRS4-ASI & High/low & $0.604(0.433-0.843)$ & $0.003 *$ & - & - \\
\hline
\end{tabular}

Notes: $* P<0.05$. - indicates no data calculated.

Abbreviations: ccRCC, clear cell renal cell carcinoma; HR, hazard ratio; Cl, confidence interval; DHRS4-ASI, DHRS4 antisense RNA I. 
A
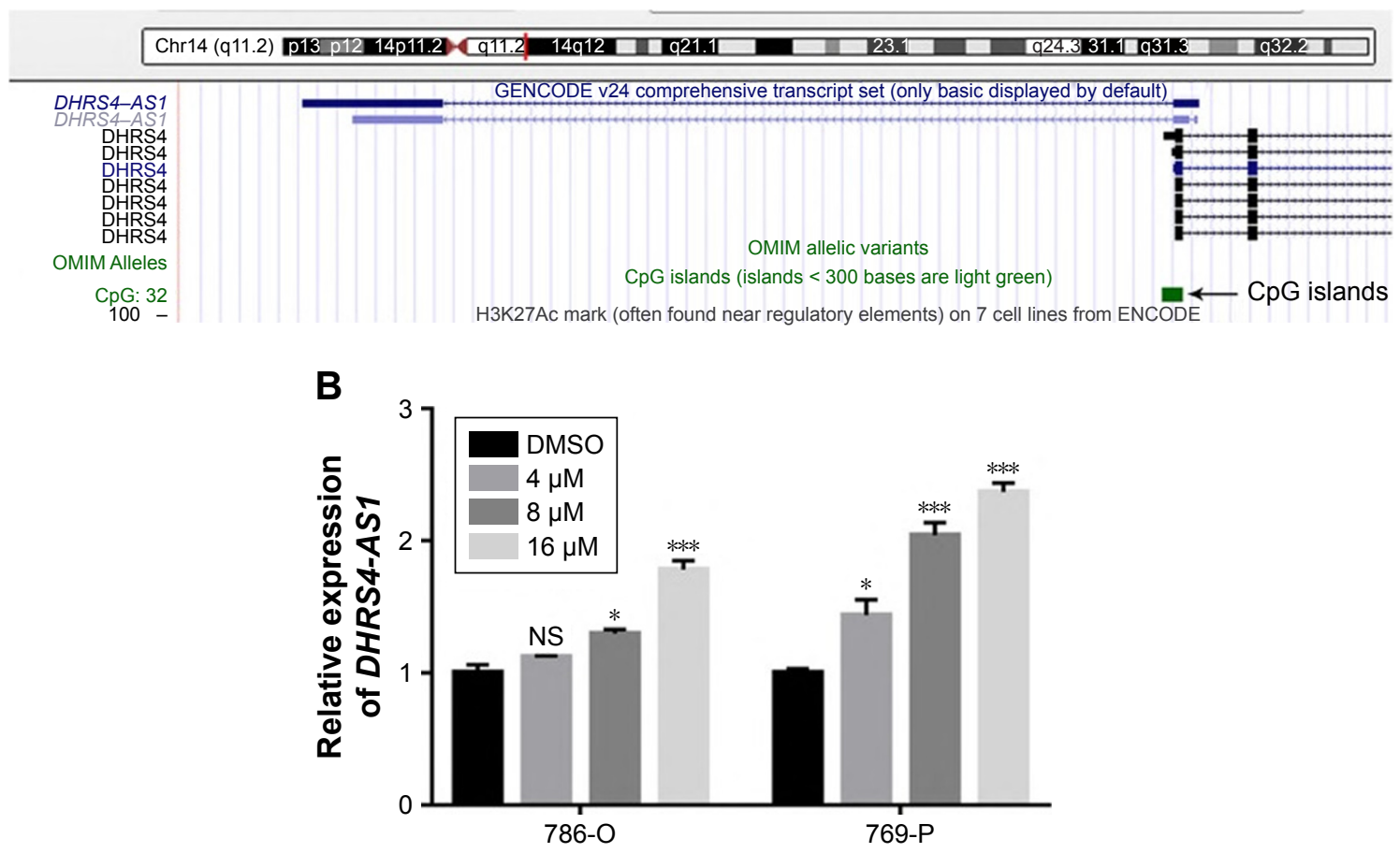

Figure SI Promoter region methylation inhibits the expression of DHRS4-ASI.

Notes: (A) The UCSC data base and find there is methylation island in the promoter region of DHRS4-ASI (green represents CpG island). (B) 786-O and 769-P cells were treated with 4, 8 and $16 \mu \mathrm{M}$ Aza and DMSO for $72 \mathrm{~h}$, expression levels of DHRS4-ASI in 786-O and 769-P cells were examined by real-time PCR. $* P<0.05$; *** $P<0.00 \mathrm{I}$. The data are presented as the mean \pm SD. Experiments were performed three times.

Abbreviations: UCSC, University of California Santa Cruz; DHRS4-ASI, DHRS4 antisense RNA I; CpG, cytosine phosphate guanine; PCR, polymerase chain reaction; NS, no significance.

\section{Publish your work in this journal}

OncoTargets and Therapy is an international, peer-reviewed, open access journal focusing on the pathological basis of all cancers, potential targets for therapy and treatment protocols employed to improve the management of cancer patients. The journal also focuses on the impact of management programs and new therapeutic agents and protocols on patient perspectives such as quality of life, adherence and satisfaction. The manuscript management system is completely online and includes a very quick and fair peer-review system, which is all easy to use. Visit http://www.dovepress.com/testimonials.php to read real quotes from published authors. 